\title{
CA19-9 tumor marker in comparison with CA125 in patients with gynecological diseases
}

\author{
Hayfaa S. AL-Hadithi FICM/path.; Aida R. Al-Derzi FICM/path \\ Assistant Professor-Dept. of Microbiology \& Immunology/Collage of Medicine/University of Baghdad \\ Assistant Professor-Dept. of Microbiology \& Immunology/Collage of Medicine/University of Baghdad
}

\begin{abstract}
Summary: Background: Cancer of the ovary is not common, but it causes more deaths than other female reproductive cancers. Women with ovarian cancer may have no symptoms or just mild symptoms until the disease is in an advanced stage because it is hard to detect early. A diagnostic approach based on the use of CA 125 in association with ultrasonography has been suggested for the early diagnosis of ovarian cancer.

Objective: To compare the utility of CA 19-9 in comparison to CA 125, for risk stratification and diagnostic purposes in patients with gynaecological diseases.

Patients and methods: A prospective study conducted in the period between November 2011 and January 2012, 90 blood samples (30 women with ovarian cancer, 30 polycystic ovarian syndrome (PCOS) cases and 30 healthy control) were collected to investigate their CA125 and CA19-9 serum levels by using ELISA technique.

Results: Serum CA125 were positive in (86.6\%) of ovarian cancer patients, and slightly elevated in (33.3\%) of PCOS patients. Serum CA19-9 were positive only in (66.6\%) of ovarian cancer patients. The sensitivity of CA125 was (86.7\%) while the specificity of CA19-9 was (96.7\%).

Conclusions: Serum CA125 and CA19-9 were significantly higher in ovarian cancer patients. The mean of the two markers were statistically higher among stage IV ovarian cancer patients as 104.09 and 94 (U/ml) for CA125 and CA19-9 respectively. Serum CA125 was more sensitive but less specific than CA19-9.

Keywords: CA-125, CA 19-9, Tumor markers, Ovarian cancer, PCOS
\end{abstract}

\section{Introduction:}

Cancer of the ovary is not common, but it causes more deaths than other female reproductive cancers. Women with ovarian cancer may have no symptoms or just mild symptoms until the disease is in an advanced stage because it is hard to detect early(1). Also the common symptoms of ovarian cancer are vague and similar to those observed in other benign conditions, so that most patients are diagnosed at advanced stages(2). Once ovarian cancer diagnosed, the stage of a tumor can be determined during surgery, and the doctor can tell if the cancer has spread outside the ovaries(3). There are four stages of ovarian cancer - Stage I (early disease) to Stage IV (advanced disease)(4).

Diagnosis of ovarian cancer starts with a physical examination (including a pelvic examination), a blood test (for some biomarkers), and transvaginal ultrasound(5). The diagnosis must be confirmed with surgery to inspect the abdominal cavity, take biopsies(tissue samples for microscopic analysis) and look for cancer cells in the abdominal fluid(6).

A diagnostic approach based on the use of CA 125 in association with ultrasonography has been suggested for the early diagnosis of ovarian cancer(7). However, this approach has several drawbacks including low sensitivity and specificity. Abnormal CA 125 serum levels can be found in malignancies of different origin including epithelial (endometrial, endocervix and lung cancer) and non-epithelial malignancies (lymphomas). Abnormal CA 125 serum levels may be also found in several benign diseases, mainly those with effusions, liver or renal failure and benign gynaecological conditions (ovarian cysts, polycystic ovary and endometriosis)(8). Sensitivity of CA 125 in ovarian cancer is related to tumor stage. Twenty percent of people with ovarian cancer have a normal CA-125, so it may not be all that reassuring if blood test is normal. Additionally, the CA-125 blood test can be elevated in benign conditions (endometriosis) and can change during the menstrual cycle(9).

Another tumor marker has been proposed, CA 19-9 is a mucin-glycoprotein derived from a human colorectal carcinoma cell line. It is related to the Lewis blood group protein and is present in epithelial tissue of the stomach, gall bladder, pancreas, and prostate. CA 19-9 is a tumor-associated antigen which is elevated in pancreatic cancers, cancers of the upper gastrointestinal tract, ovarian cancer, hepatocellular cancer, colorectal cancer, inflammatory conditions of the hepatobiliary system, and in thyroid diseases(10\&11).However, CA 19-9 is not specific of ovarian cancer and some expression has also been found in other malignancies, but it could be more specific as compared with CA 125 in patients with gynaecological disease (benign and malignant conditions)(12). 
The aims of this study were to compare the utility of CA 19-9 in comparison to CA 125 , for risk stratification and diagnostic purposes in patients with gynaecological diseases.

\section{Materials and method:}

A prospective study conducted in the period between November 2011 and January 2012.Thirty female patients with ovarian cancer and another thirty female patients with benign gynaecological diseases (PCOS) attending Baghdad medical city teaching hospital were included in this study, in addition 30 apparently healthy fertile women whose age was matched with the patients groups and ranging from (19-50) years.

The group with malignant disease (ovarian cancer), classified according to the International Federation of Gynaecology and Obstetrics. PCOS cases diagnosed according to the criteria confirmed by European society of human reproductivity and embryology and American society for reproductive medicine(4).

Blood samples were obtained from each individual by venous puncture, then was left to clot at room temperature, centrifuged and serum was collected for the detection of serum CA-125 (RayBio:Human CA125 ELISA Kit) and CA19-9 (RayBio:Human CA19-9 ELISA Kit) through application of sandwich Enzyme linked immunosorbent assay (ELISA) technique. We have considered $35 \mathrm{U} / \mathrm{ml}$ and $40 \mathrm{U} / \mathrm{mi}$ as the upper limits of normality for CA-125 and CA 19-9 respectively.

Statistical analysis was performed, the results were expressed as mean and SD. The value of $(\mathrm{p}<0.05)$ was considered statistically significant. Efficacy of each tumor marker in ovarian cancer were calculated according to its sensitivity, specificity, positive predictive value and negative predictive value.

\section{Results:-}

The differences in mean of serum CA19-9 level (U/ml) and serum CA125 level (U/ml) among included study groups were shown in table (1).The mean values of CA125 and CA19-9 were significantly higher among ovarian cancer patients in comparison with that in PCOS and healthy women $(P$ value $<0.001,<0.05$ respectively).

Table (1): The differences in mean of serum CA19-9 level (U/ml) and serum CA125 level (U/ml) among included study groups

\begin{tabular}{|c|c|c|c|c|}
\hline & \multicolumn{3}{|c|}{ Study group Number=90 } \\
\hline Values & Ovarian cancer Patient & PCOS patients & Health group \\
\hline Number & 30 & 30 & 30 \\
\hline $\begin{array}{c}\text { CA19-9(U/ml) } \\
\text { Mean } \pm \text { SD* }\end{array}$ & $68.83 \pm 44.6$ & $15.83 \pm 7.25$ & $10.7 \pm 5.91 \quad p<0.05$ \\
\hline $\begin{array}{c}\text { CA125(U/ml) } \\
\text { Mean } \pm \text { SD }\end{array}$ & $80.83 \pm 54.6$ & $38.9 \pm 67.05$ & $11.6 \pm 8.44 \quad p<0.001$ \\
\hline
\end{tabular}

$* \mathrm{SD}=$ standard deviation

Figure (1) shows the differences in mean of serum CA19-9 level (U/ml) and serum CA125 level (U/ml) among patients with different stages of ovarian cancer. The mean of the two markers were statistically higher among stage IV ovarian cancer patients as 104.09 and 94 (U/ml) for CA125 and CA19-9 respectively.

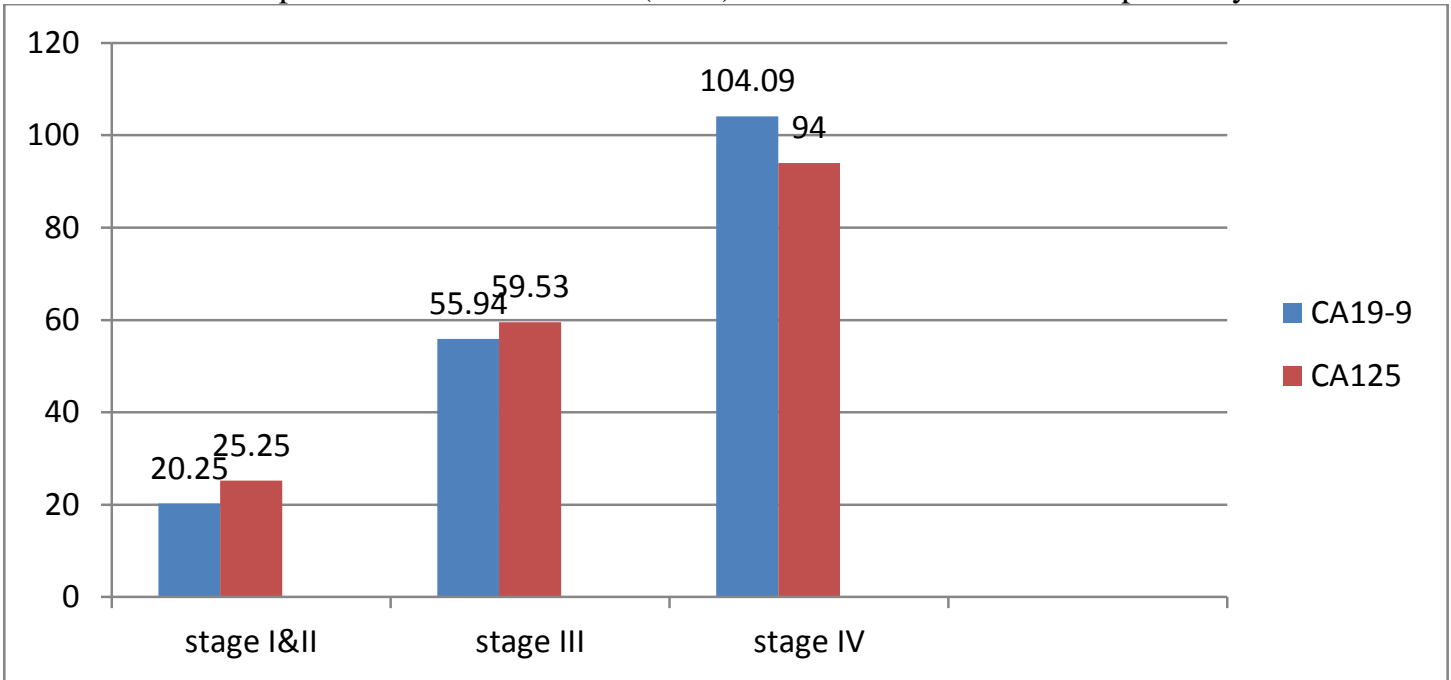

Figure (1) The differences in mean of serum CA19-9 level (U/ml) and serum CA125 level (U/ml) among patients with different stages of ovarian cancer 
The sensitivity, specificity, positive predictive value (PPV), negative predictive value (NPV) and efficacy of CA125 and CA19-9 were assayed in table (2). The highest sensitivity was for CA125 (86.7\%) while the highest specificity was for CA19-9 (96.7\%) which revealed that the efficacy of CA19-9 was more than that of CA125.

Table (2): Evaluation of CA19-9 and CA 125 efficacy in the determination of the risk of ovarian cancer (including only patients with PCOS and ovarian cancer)

\begin{tabular}{|c|c|c|c|c|c|}
\hline $\begin{array}{c}\text { Tumor } \\
\text { marker }\end{array}$ & Sensitivity & Specificity & PPV* & NPV** & Efficacy \\
\hline CA19-9 & $66.7 \%$ & $96.7 \%$ & $95.2 \%$ & $74.4 \%$ & $81.7 \%$ \\
\hline CA125 & $86.7 \%$ & $66.7 \%$ & $72.2 \%$ & $83.3 \%$ & $76.7 \%$ \\
\hline
\end{tabular}

* positive predictive value

** negative predictive value

IV. Discussion:-

Different authors have suggested the use of serial CA 125 in combination with ultrasonography in postmenopausal asymptomatic women as an aid in the early diagnosis of ovarian cancer(7,13\&14). Abnormal serum levels of CA 125 may be found in several benign and malignant diseases other than ovarian cancer(8). Despite these issues, CA 125 is used to differentiate benign from malignant pelvic masses and is used as a prognostic factor in the early diagnosis of recurrence or to assess response to treatment. It is therefore necessary to combine CA 125 with new tumor markers that provide a better diagnostic efficiency(15).

This study showed the significant increase of serum CA125 in ovarian cancer patients, and it's slight elevation in 10 out of 30 PCOS cases, it is even elevated in one healthy women. CA19-9 levels were significantly elevated in ovarian cancer patients when compared to PCOS and healthy women.

These results agreed with some previous studies which improved the use of CA125 in routine diagnosis of different gynecological problems and to differentiate benign from malignant cases(8\&16). Some studies applied on the role of CA19-9 in different malignant diseases including ovarian cancer documented it's low sensitivity when compared with hepatic or prostatic malignancies(12\&17).

Our study showed that the increased levels of the studied tumor markers were significantly higher among stage IV ovarian cancer patients than other stages, this could be due to the fact that most of our patients presented at last stages. These results agreed with those who showed the higher sensitivity of CA 125 in an advance stage of ovarian cancer in contrast to early stages of the disease $(4,9 \& 18)$.

As CA125 was elevated even in normal individual, in our study, it showed a higher sensitivity but a lesser specificity when compared with CA19-9. While CA19-9 not elevated sharply only in advanced cases of ovarian cancer. These results agree with many previous studies which documented that CA125 lack the specificity and they try to find another tumor markers which show more specificity such as HE4 $(9,12 \& 19)$.

\section{Conclusions:-}

1. Serum CA125 were significantly higher in $(86.6 \%)$ of ovarian cancer patients, and slightly elevated in $(33.3 \%)$ of PCOS patients.

2. Serum CA19-9 were positive only in (66.6\%) of ovarian cancer patients.

3. The mean of the two markers were statistically higher in advanced stages of ovarian cancer.

4. Serum CA125 was more sensitive but less specific than CA19-9 in the diagnosis of advanced cases of ovarian cancer.

\section{References:-}

[1]. Kumar R; Mishra M; Singh JK; et al. Evaluation of the Significance of CA-125 Levels in Patients With Epithelial Ovarian Cancer in Bihar, India. Clinical Ovarian Cancer: Vol 2( 2), November 2009: 118-120

[2]. McIntosh M, Anderson G, Drescher C, et al. Ovarian cancer early detection claims are biased. Clin Cancer Res 2008;14:7574.

[3]. Jacobs IJ, Menon U. Progress and challenges in screening for early detection of ovarian cancer. Mol Cell Proteomics. 2004;3:355366.

[4]. Rice GE, Edgell TA, Autelitano DJ. Evaluation of midkine and anterior gradient 2 in a multimarker panel for the detection of ovarian cancer. J Exp Clin Cancer Res 2010;29:62.

[5]. Palmer C, Duan X, Hawley S, et al. Systematic evaluation of candidate blood markers for detecting ovarian cancer. PLoS One 2008;3:2633.

[6]. Anderson GL, McIntosh M, Wu L, et al. Assessing lead time of selected ovarian cancer biomarkers: a nested case-control study. J Natl Cancer Inst 2010;102:26-38.

[7]. Su F, Lang J, Kumar A, et al. Validation of candidate serum ovarian cancer biomarkers for early detection. Biomark Insights 2007;2:369-75.

[8]. Greene MH, Feng Z, Gail MH. The importance of test positive predictive value in ovarian cancer screening. Clin Cancer Res 2008;14:7574. 
[9]. Havrilesky LJ, Whitehead CM, Rubatt JM, et al. Evaluation of biomarker panels for early stage ovarian cancer detection and monitoring for disease recurrence. Gynecol Oncol 2008;110:374-82

[10]. Cramer DW, Bast RC, Berg CD, et al. Ovarian Cancer Biomarker Performance in Prostate, Lung, Colorectal, and Ovarian Cancer Screening Trial Specimens. Cancer Prevention 2011.

[11]. Lee E. Moore; Ruth M. Pfeiffer; Zhen Zhang et al. Proteomic Biomarkers in Combination with CA-125 for Detection of Epithelial Ovarian Cancer Using Prediagnostic Serum Samples from the Prostate, Lung, Colorectal and Ovarian (PLCO) Cancer Screening Trial. Cancer vol.118(1). January 2012.

[12]. Bast RC, Jr, Klug TL, Schaetzl E, Lavin P, Niloff JM, Greber TF, et al. Monitoring human ovarian carcinoma with a combination of CA 125, CA 19-9, and carcinoembryonic antigen. Am J Obstet Gynecol. 1984;149:553-559.

[13]. Bast RC, Jr, Badgwell D, Lu Z, Marquez R, Rosen D, Liu J, et al. New tumour markers: CA 125 and beyond. Int J Gynecol Cancer. 2005;15(Suppl 3):274-281.

[14]. Duffy MJ, Bonfrer JM, Kulpa J, Rustin GJ, Soletormos G, Torre GC, et al. CA 125 in ovarian cancer: European Group on Tumour Markers guidelines for clinical use. Int J Gynecol Cancer. 2005;15:679-691.

[15]. Moore RG, Maclaughlan S, Bast RC., Jr Current state of biomarker development for clinical application in epithelial ovarian cancer. Gynecol Oncol. 2010;116:240-245.

[16]. Sturgeon CM, Duffy MJ, Stenman UH, Lilja H, Brunner N, Chan DW, et al. National Academy of Clinical Biochemistry laboratory medicine practice guidelines for use of tumour markers in testicular, prostate, colorectal, breast, and ovarian cancers. Clin Chem. 2008;54:11-79.

[17]. Engelen MJ, de Bruijn HW, Hollema H, et al. Serum CA 125, carcinoembryonic antigen, and CA 19-9 as tumor markers in borderline ovarian tumors. Gynecol Oncol 2000;78:16-20.

[18]. Anastasi E, Marchei GG, Viggiani V, Gennarini G, Frati L, Reale MG. HE4: a new potential early biomarker for the recurrence of ovarian cancer. Tumour Biol 2010;31:113-9.

[19]. Huhtinen K, Suvitie P, Hiissa J, et al. Serum HE4 concentration differentiates malignant ovarian tumours from ovarian endometriotic cysts. Br J Cancer 2009;100:1315-9. 